\title{
Medically unexplained symptoms: assessment and management
}

\author{
Authors: Mujtaba Husain ${ }^{A}$ and Trudie Chalder ${ }^{B}$
}

\begin{abstract}
Medically unexplained symptoms or persistent physical symptoms are common, real and are associated with significant distress, loss of functioning and high healthcare costs. History, examination and appropriate investigations are essential to make a diagnosis. Once the diagnosis has been made, exploring the impact of the symptoms helps us to tailor our advice to patients. This paper sets out a practical approach to taking a history, assessment and stepwise management principles.
\end{abstract}

KEYWORDS: cognitive behavioural therapy, medically unexplained symptoms, persistent physical symptoms

DOI: $10.7861 /$ clinmed.2020-0947

\section{Introduction}

Medically unexplained symptoms (MUS) or persistent physical symptoms (PPS) are very common. They account for up to one in five presentations in primary care. ${ }^{1,2}$ This can be even higher in some secondary care clinics. ${ }^{3}$ Hence assessing and supporting people with unexplained symptoms is a significant part of the everyday work of most clinicians.

PPS are often associated with significant distress, impaired functioning, loss of role and high use of healthcare and welfare. ${ }^{4}$ Patients often report feeling that their concerns are not taken seriously by their healthcare professionals. ${ }^{5}$ Doctors also report feeling unprepared for helping people with PPS. ${ }^{6}$ PPS are also estimated to account for approximately $10 \%$ of total $\mathrm{NHS}$ expenditure for the working-age population in England.?

Much of this delivers limited value to the patient and can be counterproductive or even harmful (for example, multiple or repeat investigations) ${ }^{8}$

The longer the duration of untreated symptoms the poorer the prognosis..$^{9,10}$ Evidence-based treatment is available. ${ }^{9,11}$ Yet, it is common for diagnosing, explaining the diagnosis to the patient and offering treatment to be delayed. 5

Authors: ${ }^{A}$ consultant liaison psychiatrist, South London and Maudsley NHS Foundation Trust (SLaM), London, UK; Bprofessor of cognitive behavioural psychotherapy, King's College London, London, UK and South London and Maudsley NHS Foundation Trust (SLaM), London, UK

\section{Definitions and classification: one syndrome or many?}

The language we use to describe PPS can be complex and confusing. Adjectives such as functional, somatic, unexplained and dissociative are commonly used. And there are specialtyspecific diagnoses like irritable bowel syndrome, fibromyalgia and functional neurological disorder. Almost every medical specialty has an equivalent diagnostic category.

It has been proposed that PPS can be considered as one syndrome rather than many. ${ }^{12}$ There is significant overlap in the risk factors and symptoms seen in specific diagnoses..$^{13}$ PPS frequently present with multiple symptoms that cut across specialty boundaries (which can result in multiple referrals). A number of symptoms (such as fatigue, pain and disrupted sleep) are common across diagnoses. Indeed, many of the challenges of helping people with PPS stem from a fragmented approach to management where the symptoms cut across medical silos and divides (primary and secondary care; physical and mental health; and different medical specialties).

The Diagnostic and Statistical Manual of Mental Disorders, 5th edn (DSM-5) now defines somatic symptom disorder. ${ }^{14}$ Under DSM-IV, symptoms had to be unexplained but the new definition removes this requirement and focuses on distress and disruption of daily life. International Classification of Diseases, 11th revision (ICD-11) proposes the similar bodily distress disorder.

The term PPS has also been shown to rank highly in surveys of patient preference. ${ }^{15,16}$ MUS and PPS describe similar symptoms but are not quite the same thing. Describing symptoms as medically unexplained can be seen by patients as dismissive. ${ }^{17}$ It has been suggested PPS should occupy a neutral space with neither a somatic disease aetiology, nor a mental disorder to reflect their complex nature (analogous to pain disorders in ICD-11). ${ }^{18,19}$

\section{Physical and mental health}

Having a physical health diagnosis increases the risk of experiencing unexplained symptoms. For example, around a fifth of those diagnosed with non-epileptic seizures also have epilepsy, non-cardiac chest pain is common after myocardial infarction and dysfunctional breathing is frequently seen in those with asthma. ${ }^{20-22}$ Hence, the divide between explained and unexplained symptoms is not quite as clear cut as the terms imply. 
Table 1. Taking a history

Task

Elicit symptoms

Triggers

Physical and mental health screening

Impact

Sleep

\section{Current} medication

Past medical and psychiatric history

Family history

\section{Suggested questions and approach}

Start with open questions to elicit symptoms: Tell me about the problems or symptoms?

Where there are many symptoms, by asking which symptom or symptoms are the worst can help to focus a history (eg 'If you could take one symptom away which would it be?')

Then move to more specific questions

$>$ What and where are the symptoms?

$>$ How long have they had them?

$>$ When do they occur?

$>$ Do they fluctuate?

$>$ What else happens?

$>$ Are there associated symptoms?

$>$ What brings them on?

$>$ What makes them better?

$>$ What makes them worse?

Ask about commonly seen associated symptoms: fatigue, pain and poor sleep.

Do they avoid things that could bring on the symptoms?

Is there a boom and bust pattern to the symptoms?

Did anything bring these symptoms on?

Did you have any illnesses or health problems when the symptoms first came on?

Were there any stressful things or big changes in your life around the time the symptoms started?

Ask about red flag symptoms as you would do for anyone with physical symptoms.

Ask about depression, anxiety and stress: How has this affected your mood?

If their mood is low: Which do you think came first?

It's common for people with physical symptoms like yourself to feel low or worried; has that happened with you?

How has this affected you?

Are there things you've had to stop doing?

Think about the impact on different areas of life.

$>$ Work/education

$>$ Social life and relationships

$>$ Home life, everyday tasks and activities of daily living

> Mobility and limits on what they can do? At the most severe end, are they largely housebound or even bedbound?

Asking the patient to describe a typical day can be helpful, particularly to get a sense of what they are actually doing.

Significant others; do they have a caring role? Do they have other support? Are there any potential reinforcing factors?

Has this affected their sleep? Detail here can help with the advice you give on sleep hygiene later.

$>$ What time they go to bed and time they fall asleep.

$>$ The number of times they wake up in the night and how long.

$>$ What time they wake up in the morning and time they get up.

$>$ If they take daytime naps.

Both prescribed and non-prescribed/over-the-counter medication.

Are they using any regular pain relief?

Long-term conditions and past illness.

Childhood illness.

History of mental health problems.

Also ask about interventions for their physical symptoms: cognitive behavioural therapy or other talking therapies, medication, medical procedures and alternative treatments.

What has been tried so far?

Did it help?

Has anyone in your family had similar symptoms?

Has anyone in your family had other physical symptoms that lasted a long time?

Ask if there are any other long-term conditions or mental health conditions in close family members, including things that occurred in the patient's childhood. 
Table 1. Taking a history (Continued)

Task

\section{Suggested questions and approach}

Social history

Employment, education, housing/living arrangements, welfare and benefits.

Drugs and alcohol

Is there any use of alcohol or recreational drugs, particularly to help alleviate the symptoms?

Personal Are opiate painkillers being used, either prescribed or non-prescribed?

history Childhood adversity/illness, parental ill health, history of traumatic events and attitude to illness in family.

Mental health comorbidity is also common. Around $50 \%$ of individuals with PPS suffer from associated anxiety or depression, which is more than that seen in other long-term conditions. ${ }^{3,23}$ This may be due to the impact of living with persistent symptoms and the distress and impairment they cause, it may reflect a common aetiology, or it may be a combination of the two.

Associated physical and mental health problems stress the need for a thorough assessment to ensure the treatment approach offered addresses comorbidity.

\section{Aetiology}

The most convincing models for how PPS develop take a multifactorial approach. Deary, Chalder and Sharpe have described a cognitive behavioural model of persistent physical symptoms and how they develop 9 .

Some factors which increase the risk of developing PPS are nonmodifiable. These include parental ill health during childhood; illness during childhood; childhood adversity and abuse; personality traits; and having a long-term condition and a family history of someone with a long-term condition.

There can often be a 'trigger' (but this is not always the case). This could be an infection, a physical illness or trauma. It may be a psychosocial stressor or repeated stressors. Often both physical and psychosocial stressors precede the onset of PPS.

Then there are maintaining factors which keep the symptoms going. These can be divided into four groups.

> Physiological: autonomic dysregulation, central sensitisation, hypothalamic-pituitary-adrenal (HPA) axis dysfunction and sleep disruption.

$>$ Social: loss of role and medical uncertainty.

> Cognitive: catastrophic misappraisal of symptoms, symptom focusing and intolerance of uncertainty.

> Behavioural: avoidance behaviour, 'all or nothing' behaviours and poor sleep hygiene.

Treatment focuses on addressing modifiable maintaining factors, particularly cognitive and behavioural factors.

Another model describes the brain as actively making predictions or inferences rather than passively waiting for sensory input from the body. It proposes PPS are due to 'failures of inference' in which expectations, cognition and emotion play a part in symptom perception ${ }^{24}$

It is now recognised that a variety of physiological processes are involved in PPS. There is increasing evidence that autonomic nervous system dysregulation plays a role. For example, a recent meta-analysis found differences in heart rate variability comparing patients with PPS to healthy controls. ${ }^{25}$ Central sensitisation has also been proposed as a common physiological basis for PPS. ${ }^{26}$ Other hypotheses include immune dysfunction and metabolic abnormalities. ${ }^{18}$

\section{Assessment}

As ever in medicine, assessment of PPS starts with a thorough history. Active listening with empathy is important to help the patient feel heard and understood. Table 1 outlines a suggested approach and key questions as you do this.

Physical examination (cardiovascular, respiratory, abdominal and neurological examinations) is essential to look for signs of other physical health problems. In some functional neurological disorders, there are also positive signs that can be elicited. ${ }^{27}$

Baseline physical observations (heart rate, blood pressure and temperature) are required.

The following investigations should be undertaken as a minimum:

> urinalysis for protein, blood and glucose

$>$ full blood count

$>$ urea and electrolytes

$>$ liver function

$>$ thyroid function

> erythrocyte sedimentation rate and C-reactive protein

$>$ random blood glucose

$>$ serum creatinine

> tissue transglutaminase antibodies (tTG-IgA) for coeliac disease

$>$ serum calcium

$>$ creatine kinase.

Further investigations may be indicated. For example, for persistent unexplained gastrointestinal symptoms in women, particularly for those aged over 50 years, CA125 should be measured..$^{28}$

Common diagnoses which are sometimes missed include diabetes, coeliac disease, thyroid problems and inflammatory conditions.

While diagnoses of persistent physical symptoms should be made carefully only after a full assessment, evidence now suggests that when a diagnosis is made, they are stable diagnoses with low misdiagnosis rates. ${ }^{29}$ Doctors are more likely to under-diagnose than over-diagnose unexplained symptoms. ${ }^{30}$

\section{Management}

Treatment takes a stepwise approach.

\section{Positive diagnosis and explanation}

The first and most important step is a positive diagnosis and explanation of what the diagnosis means. This must be a 
Table 2. Explaining the diagnosis

Task

Make a positive diagnosis

Explain it is common and give them hope about treatment

Tell them you believe them

Tell them what we know about persistent physical symptoms and be honest about what we don't know

Say something about what we do know of the biology and mind-body interaction

\section{Suggested phrases}

These symptoms and test results tell us you have fibromyalgia / non-cardiac chest pain / persistent physical symptoms etc (depending on the presentation).

In my clinic around one in five people I see have problems similar to yours. People can, and do, get better with time.

There are things we can work on and treatments that can help.

Your symptoms are real, and I believe you.

It is normal to have real physical symptoms that don't show up on blood tests.

These conditions tend to have many causes.

We know there are many risk factors that make people more likely to have these problems.

We know there are clear physical factors that are part of this. For example, infections or illnesses can often be the trigger. But the symptoms can keep going even when the infection/virus is long gone. We know there can be physiological changes; sleep rhythms are often affected and there can be hormonal changes.

The mind and the body work together, particularly in complex conditions like these. We can't think about one without the other. For example, when we are anxious our heart beats faster and we can feel anxiety physically in our stomachs. two-way conversation and should be tailored to the individual's concerns and experiences. Ask what they think may be the cause and what they are worried about. Answer their questions and engage in a dialogue. It is crucial that every clinician, whether in primary care, the emergency department or secondary care sees this as part of their role. Table 2 sets out a guide and suggested phrases.

\section{Guided self-help}

For those presenting for the first time, particularly with a shorter duration of symptoms and less functional impairment, a guided self-help approach is the next step.

Direct them to useful resources like www.nhs.uk/conditions/ medically-unexplained-symptoms and www.rcpsych.ac.uk/mentalhealth/problems-disorders/medically-unexplained-symptoms.
There is more specific information and support available for some presentations, for example from https://fndhope.org for functional neurological symptoms.

Approaches at this stage include:

> advice on managing activity (Table 3)

$>$ advice on stress management

$>$ improving sleep hygiene particularly:

$>$ a consistent bedtime and wake up time

> if they have difficulty falling asleep, getting up for 30 minutes to do something to distract themselves out of the bedroom environment

$>$ reducing daytime naps

$>$ tailored advice on general health, diet and exercise

$>$ treating associated depression or anxiety, considering medication if appropriate.

\section{Table 3. Managing activity}

Task

Explore the impact of the symptoms

Decide what to work on

Agree on goals to increase enjoyable or valued activities

Discussion if they are overdoing things

\section{Suggested approach and phrases ${ }^{31}$}

What do these symptoms stop you from doing?

What would you like to be able to do more of?

Are there things you enjoy that you've had to cut out or avoid?

Discuss how increasing enjoyable or valued activities can help.

Choose one or two areas to focus on.

Make these achievable and specific (what, when, where and with whom).

Using a SMART approach can be useful.

Start today and review at next appointment (eg 2 weeks in primary care).

Let people know symptoms can sometimes get a bit worse before they get better.

Consistency is important, whether it is a good or bad day with the individual's symptoms.

Little and often is better than all or nothing.

Do you think it could help to manage your activities in a different way?

SMART = specific, measurable, achievable, realistic and timely. 
We should not fall into the trap of referring patients for therapy or specialist care while skipping the first essential steps of making a positive diagnosis, giving a positive explanation and guiding patients through self-help. Many patients will not need further referral when these steps are taken.

\section{Cognitive behavioural therapy}

If things are not improving after $4-8$ weeks the next step is offering cognitive behavioural therapy (CBT). CBT is the treatment with the best evidence base for persistent physical symptoms, both for general symptoms and more specific syndromes, and overall there is evidence for a moderate beneficial effect. . $^{911,32}$ CBT is available through local IAPT (Improving Access to Psychological Therapy) services where patients can also self-refer.

When offering CBT, it can be helpful to say that it has been shown to help people with physical symptoms in long-term physical health conditions like multiple sclerosis; CBT is a treatment which has the potential to help whatever the cause of PPS. ${ }^{33}$

\section{Specialist referral}

Where first-line CBT has not helped, specialist referral should be considered. In practice, what is available can vary depending on where you are based and the symptoms the individual has. However, a multidisciplinary approach is best. Good communication and consistency between healthcare professionals around the explanations given and the plan is essential. Research suggests psychological treatments are helpful in terms of improving symptoms and alleviating their impact, and so this message can be delivered with some confidence.

Specialist referral should be considered earlier if employment or education is at risk; there is physical or mental health comorbidity; particularly frequent attendance to emergency departments; or multiple specialists are already involved.

In some cases, harm minimisation is also important. Repeat investigations and interventions can lead to direct and indirect iatrogenic harm. Involving all healthcare professionals and/or departments the patient attends and discussing a plan to limit or stop harmful interventions with the patient is essential. For example, this might be a multidisciplinary plan on what happens in the emergency department or regarding invasive investigations.

\section{Funding}

Trudie Chalder is part-funded by the National Institute for Health Research (NIHR) Biomedical Research Centre at South London and Maudsley NHS Foundation Trust, and King's College London at King's College Hospital NHS Foundation Trust.

\section{References}

1 de Waal MW, Arnold IA, Eekhof JA, van Hemert AM. Somatoform disorders in general practice: prevalence, functional impairment and comorbidity with anxiety and depressive disorders. British Journal of Psychiatry 2004;184:470-6.

2 Peveler R, Kilkenny L, Kinmonth AL. Medically unexplained physical symptoms in primary care: a comparison of self-report screening questionnaires and clinical opinion. J Psychosom Res 1997;42: 245-52.

3 Nimnuan C, Hotopf M, Wessely S. Medically unexplained symptoms: An epidemiological study in seven specialities. J Psychosom Res 2001;51:361-7.
4 Dirkzwager AJ, Verhaak PF. Patients with persistent medically unexplained symptoms in general practice: characteristics and quality of care. BMC Fam Pract 2007;8:33.

5 Chew-Graham CA, Heyland S, Kingstone T et al. Medically unexplained symptoms: continuing challenges for primary care. $\mathrm{Br}] \mathrm{Gen}$ Pract 2017:67:106-7.

6 Yon K, Nettleton S, Walters K et al. Junior doctors' experiences of managing patients with medically unexplained symptoms: a qualitative study. BMJ Open 2015;5:e009593.

7 Bermingham SL, Cohen A, Hague J, Parsonage M. The cost of somatisation among the working-age population in England for the year 2008-2009. Ment Health Fam Med 2010;7:71-84.

8 The King's Fund. Bringing together physical and mental health: A new frontier for integrated care. The King's Fund, 2016. www. kingsfund.org.uk/publications/physical-and-mental-health [Accessed 01 November 2020].

9 Deary V, Chalder T, Sharpe M. The cognitive behavioural model of medically unexplained symptoms: A theoretical and empirical review. Clin Psychol Rev 2007;27:781-97.

10 Jackson JL, Passamonti M. The outcomes among patients presenting in primary care with a physical symptom at 5 years. J Gen Intern Med 2005;20:1032-7.

11 van Dessel N, den Boeft M, van der Wouden JC et al. Nonpharmacological interventions for somatoform disorders and medically unexplained physical symptoms (MUPS) in adults. Cochrane Database Syst Rev 2014:CD011142.

12 Wessely S, Nimnuan M, Sharpe M. Functional somatic syndromes: one or many. Lancet 1999;354:936-9.

13 Chalder T, Willis C. "Lumping" and "splitting" medically unexplained symptoms: is there a role for a transdiagnostic approach? J Ment Health 2017:26:187-91.

14 Dimsdale JE, Levenson J. What's next for somatic symptom disorder? Am J Psychiatry 2013;170:1393-5.

15 Picariello F, Ali S, Moss-Morris R, Chalder T. The most popular terms for medically unexplained symptoms: the views of CFS patients. J Psychosom Res 2015;78:420-6.

16 Marks EM, Hunter MS. Medically unexplained symptoms: an acceptable term? Br J Pain 2015;9:109-14.

17 Stone J, Wojcik W, Durrance D et al. What should we say to patients with symptoms unexplained by disease? The 'number needed to offend'. BMJ 2002;325:1449-50.

18 Burton C, Fink P, Henningsen P, Löwe B, Rief W. Functional somatic disorders: discussion paper for a new common classification for research and clinical use. BMC Med 2020;18:34.

19 Treede RD, Rief W, Barke A et al. Chronic pain as a symptom or a disease: the IASP classification of chronic pain for the international classification of diseases (ICD-11). Pain 2019;160:19-27.

20 Mellers JD. The approach to patients with "non-epileptic seizures". Postgrad Med J 2005;81:498-504.

21 Qintar M, Spertus JA, Tang Y et al. Noncardiac chest pain after acute myocardial infarction: frequency and association with health status outcomes. Am Heart J 2017;186:1-11.

22 Boulding R, Stacey R, Niven R et al. Dysfunctional breathing: a review of the literature and proposal for classification. Eur Respir Rev 2016:25:287-94.

23 Hotopf M, Mayou R, Wadsworth M, Wessely S. Temporal relationships between physical symptoms and psychiatric disorder: Results from a national birth cohort. Br J Psychiatry 1998;173:255-61.

24 Henningsen P, Gündel H, Kop WJ et al. Persistent physical symptoms as perceptual dysregulation: a neuropsychobehavioral model and its clinical implications. Psychosom Med 2018;80:422-31.

25 Vreijling SR, Troudart Y, Brosschot JF. Reduced heart rate variability in patients with medically unexplained physical symptoms: a metaanalysis of HF-HRV and RMSSD. Psychosom Med 2021;83:2-15.

26 Bourke JH, Langford RM, White PD. The common link between functional somatic syndromes may be central sensitisation. J Psychosom Res 2015;78:228-36. 
27 Cock, HR, Edwards MJ. Functional neurological disorders: acute presentations and management. Clin Med 2018;18:414-7.

28 National Institute for Health and Care Excellence. Ovarian cancer: recognition and initial management: Clinical guideline [CG122]. NICE, 2011. www.nice.org.uk/guidance/cg90 [Accessed 01 November 2020].

29 Stone J, Carson A, Duncan R et al. Symptoms unexplained by organic disease in 1144 new neurology outpatients: how often does the diagnosis change? Brain 2009;132:2878-88.

30 Nimnuan C, Hotopf M, Wessely S. Medically unexplained symptoms: How often and why are they missed? QJM 2000;93:21-8

31 Chalder T, Patel M, James $\mathrm{K}$ et al. Persistent physical symptoms reduction intervention: a system change and evaluation in secondary care (PRINCE secondary) - a CBT-based transdiagnostic approach: study protocol for a randomised controlled trial. BMC Psychiatry 2019;19:307.
32 Pourová M, Klocek A, Rihacek T, Ćevelíček M. Therapeutic change mechanisms in adults with medically unexplained physical symptoms: A systematic review. J Psychosom Res 2020;134:110124.

33 van den Akker LE, Beckerman H, Collette EH et al. Cognitive behavioral therapy positively affects fatigue in patients with multiple sclerosis: results of a randomized controlled trial. Mult Scler ] 2017;23:1542-53.

Address for correspondence: Dr Mujtaba Husain, Persistent Physical Symptoms Research and Treatment Unit, King's College London, Mapother House, Denmark Hill, London SE5 8AZ, UK.

Email:mujtaba.husain@slam.nhs.uk

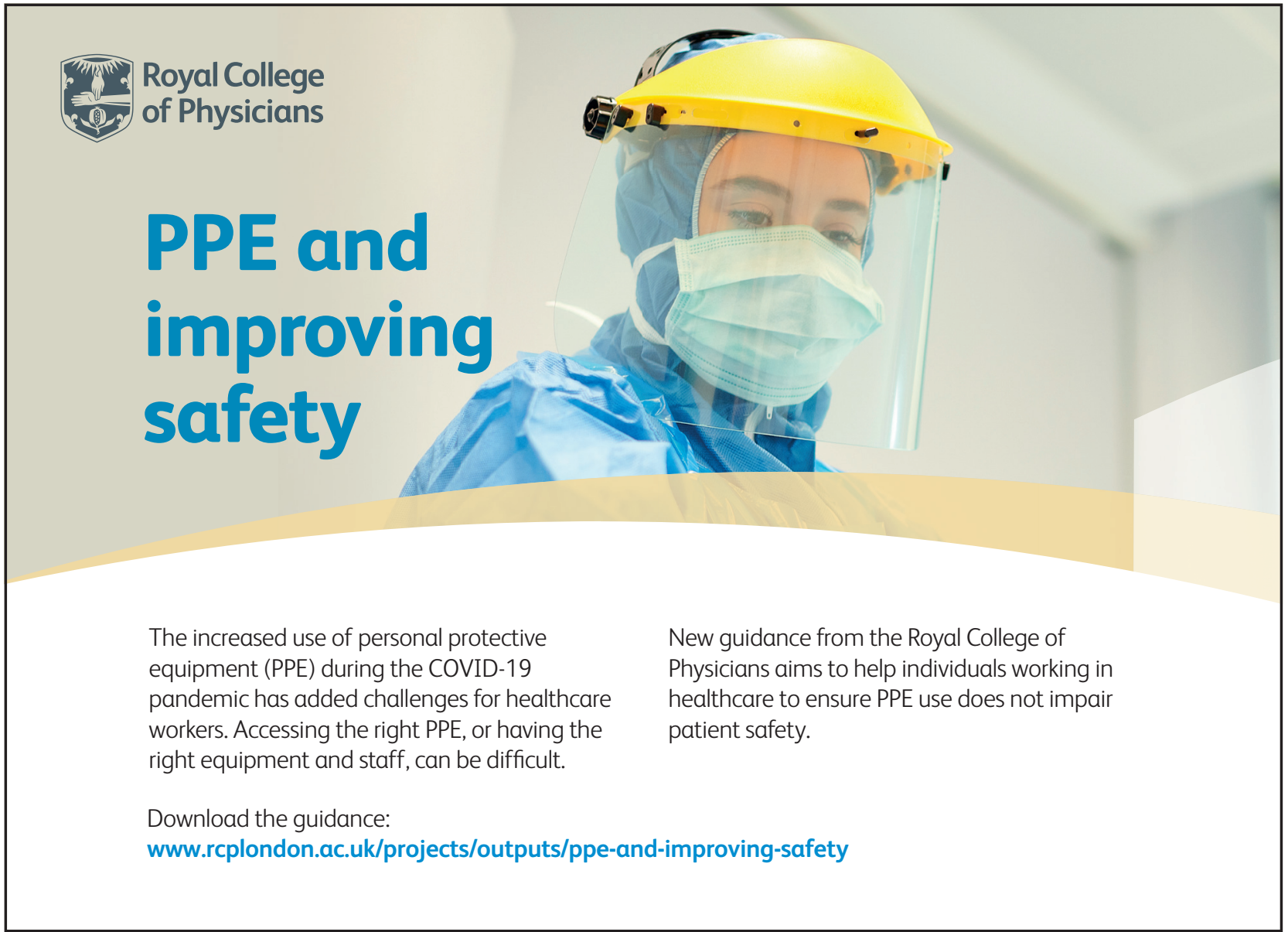

